\title{
Timing stabilization of solid-state, Yb-based laser system
}

\author{
Stefano Valente, ${ }^{1,2}$ Anne-Laure Calendron, ${ }^{1,3}$ Joachim Meier, ${ }^{1}$ Emma Kueny, ${ }^{1,2}$ \\ Hüseyin Çankaya, ${ }^{1,2,3}$ Nicholas H. Matlis, ${ }^{1}$ Giovanni Cirmi, ${ }^{1,3}$ Franz Kärtner, ${ }^{1,2,3}$ \\ ${ }^{1}$ Center for Free-Electron Laser Science, Deutsches Elektronen Synchrotron (DESY) Notkestrasse 85, 22607 \\ Hamburg, Germany \\ ${ }^{2}$ Department of Physics, University of Hamburg, Luruper Chaussee 149, 22761 Hamburg, Germany \\ ${ }^{3}$ The Hamburg Centre for Ultrafast Imaging, University of Hamburg, Luruper Chaussee 149, 22761 Hamburg, \\ Germany \\ Author's email address : stefano.valente@cfel.de
}

\begin{abstract}
We present a tight optical timing stabilization to 10 fs RMS jitter between two compressed branches of a sub-picosecond $\mathrm{Yb}$ based laser system operating at $1 \mathrm{kHz}$.
\end{abstract}

OCIS codes: (140.3615) Lasers, ytterbium; (120.3940) Metrology; (320.7100) Ultrafast measurements

\section{Introduction}

The understanding of complex physical processes on ultra-short time scales, such as strong-field physics, and the design of novel optical sources, like spectrally combined OPCPAs [1], rely on complex laser systems and tight synchronization is often required between multiple optical pulse trains. Timing synchronization has been demonstrated in laser systems such as fiber based systems for timing links to sub-fs level at $\mathrm{MHz}$ repetition rate [2,3] and at low repetition rate for the fs short pulses out of the channels of a waveform synthesizer to 250 as [4]. These systems were limited in average power. To scale the energy of a laser system or of an OPCPA or of an OPCPA synthesizer, it is necessary to use complex amplification chains relying on different laser media. These, like $\mathrm{Yb}$ doped materials, sustain lower bandwidth, increasing the pulse duration to the picosecond level. Amplification to high energies requires then long stretching ratios, long compressors and long optical paths susceptible to introduce timing jitter. In contrary to the synchronization of the outputs of an OPCPA synthesizer or on timing links, the level of synchronization required for the OPCPA pumps is in the order of $\sim 10 \mathrm{fs}$; the challenge stems from the fast and large variations of the noise. Our low repetition rate $(1 \mathrm{kHz})$, high energy laser system is based on Yb:KYW and Yb:YAG amplifiers delivering subpicosecond to few picosecond pulse duration [5-7]. In this work, we demonstrate a timing synchronization operating at $1 \mathrm{kHz}$ for sub-picosecond to picosecond long high-energy pulses.

\section{Experimental Setup}

Our experimental setup, shown in Fig.1, entails several components and is described in details in [5-7]. First, a $\mathrm{Yb}: \mathrm{KYW}$ oscillator sends $\sim 200$ fs long pulses into a stretcher based on chirped fiber Bragg gratings. The pulses stretched to $\sim 3.4 \mathrm{~ns}$ are sent to a dual-crystal, Yb:KYW regenerative amplifier operating at $1 \mathrm{kHz}$ [5].

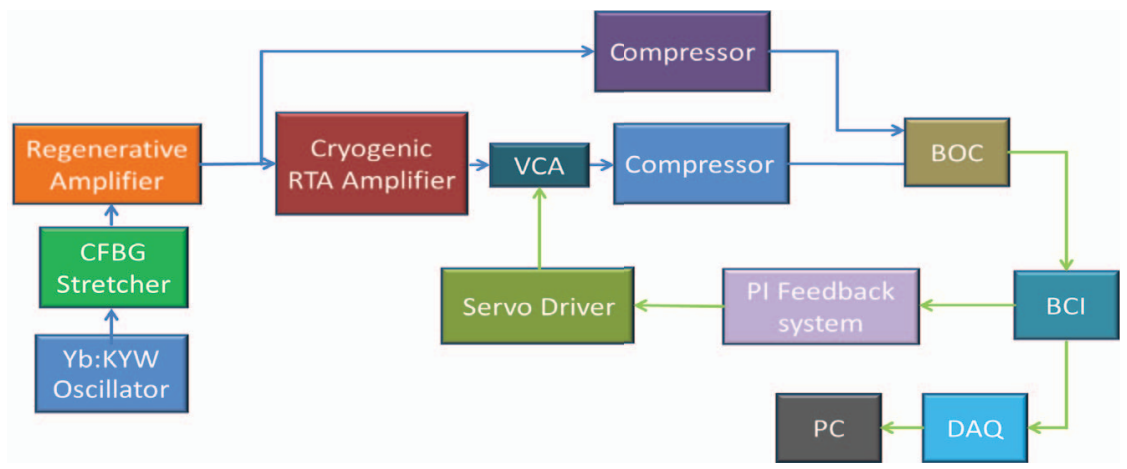

Fig. 1: Timing stabilization setup. RTA: rod type amplifier, CFBG: chirped fiber Bragg gratings, BOC: balanced optical cross correlator, BCI: box car integrator, DAQ: data aquisition, PC: personal computer, VCA: voice coil actuator. 
The regen output is split between two paths, one for direct compression, the other for future amplification inside a cryogenically cooled rod-type amplifier and compression. The optical path between the splitting and combining points is $\sim 35 \mathrm{~m}, \sim 15 \mathrm{~m}$ of it within the 2 deck compressors. The two beams with orthogonal polarization are then combined with a polarising beamsplitter cube and sent to a balanced optical cross correlator (BOC) [8-9], a nonlinear optical device based on sum-frequency generation, whose output yields the timing jitter that has to be minimized. The nonlinear crystal used is a $7 \mathrm{~mm}$ long $\beta$-Barium Borate (BBO). After increasing the detector signal to noise ratio with a box car integrator (BCI), the BOC output is then sent to a feedback chain comprising a MenloSystems controller with a proportional and integral feedback action (PI), a servo driver and a voice coil actuator (VCA) with a mirror mount for imparting the mechanical movement for timing jitter compensation. We achieve a timing resolution of 5 $\mathrm{mV} / \mathrm{fs}$ with an uncertainty factor of 2. Compared with piezoelectric transducers, VCA have lower hysteresis and larger stroke which enables motion control over longer distances, eliminating the need of a motor removing slow drifts.

\section{Results}

Figure 2(a) pictures a typical spectral content of the timing jitter up to the Nyquist frequency ( $500 \mathrm{~Hz})$. It is influenced by the overall noise in the lab (acoustic and vibrations induced e.g. by vacuum pumps). As an example of the impact of vacuum pumps in the laboratory, we show two different spectra of the noise acquired during the shut-down of a turbo pump: the blue one exhibits a peak at $324 \mathrm{~Hz}$ and the red one at $95 \mathrm{~Hz}$, both encircled. The vibrations are coupled onto the nearby optical paths. It can be seen how the amplitude of the noise gets stronger towards lower frequencies. Under these typical laboratory conditions, the jitter equals $\sim 100 \mathrm{fs}$ standard deviation; to cover $3 \sigma, 90 \mu \mathrm{m}$ stroke is necessary. Figure 2(b) shows the performance of the feedback system. At the beginning of the recording, the feedback is turned on, and the fluctuations reduced from $\sim 42 \mathrm{fs}$ to $\sim 10 \mathrm{fs}$. The feedback is switched off after $400 \mathrm{~s}$. In typical laboratory conditions, our feedback loop is able to remove the noise below $50 \mathrm{~Hz}$; the remaining variations are shotto-shot variations as in Figure 2(b), which could be addressed by the implementation of more sophisticated feedback loops.
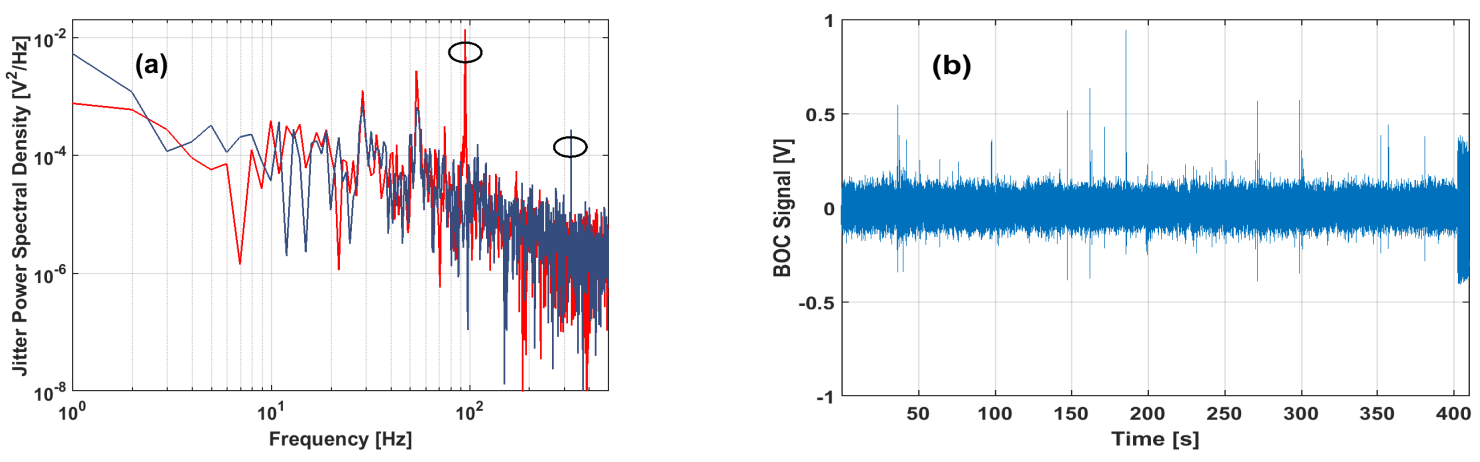

Fig. 2: (a) Jitter power spectral density. (b) Timing drift, without and with feedback loop. During this measurement, the noise in the lab was decreased due to switched off experiments

\section{Conclusion}

We have demonstrated a timing synchronization between two arms of a picosecond laser system operating at $1 \mathrm{kHz}$. We have performed an investigation of the coupling of noise sources present in the lab into our system, e.g. the noise generated by vacuum pumps. Locking to $10 \mathrm{fs}$ has been achieved with a PI feedback loop.

\section{References}

1. A. Wirth et al., Synthesized light transients. Science 334, 195-200 (2011).

2. M.Y. Peng et al. , Opt. Express 21, 19982-19989 (2013)

3. M. Xin et al., Light: Science and Applications 6, e16187 (2017)

4. S.-W. Huang et al. , Nature Photonics 5, 475-479 (2011)

5. A.-L. Calendron et al. ', Optics Express 22, 24752-24762 (2014)

6. M.Hemmer, CLEO 2015,OSA Technical Digest, STu4O.3

7. H. Çankaya et al. , Optics Express 24, 25169-25180 (2016)

8. J. Kim et al., Nature Photonics 2, 733 - 736 (2008)

9. T. R. Schibli et al., Optics Letters 28, pp. 947-949 (2003) 